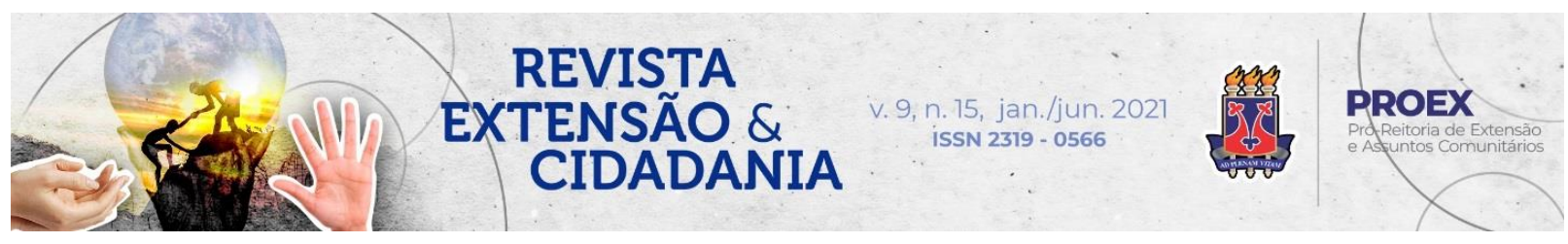

DOI: $10.22481 /$ recuesb.v9i15.8520

\title{
SAÚDE MENTAL, SÉTIMA ARTE E TERCEIRA IDADE: UM RELATO DE EXPERIÊNCIA
}

\author{
Mental health, seventh art and third age: an experience report
}

\author{
Maria Eduarda Silva Gomes Roberto ${ }^{1}$ \\ Flávio Evans Soares Brito Júnior ${ }^{2}$ \\ Gabriela Garcia de Carvalho Laguna ${ }^{3}$ \\ Luiz Henrique Pitanga Evangelista dos Santos ${ }^{4}$ \\ Ranna Santos Morais 5
}

Resumo: O evento multidisciplinar "Cine Saúde" que ocorreu no mês de abril do corrente ano 2021, trouxe a partir de uma obra cinematográfica um ponto de partida para discutir a demência. Objetivo: promover um ambiente democrático e elucidativo para promoção do diálogo ampliado sobre o envelhecimento e a demência. Metodologia: $O$ evento foi realizado em plataforma virtual (Google Meet), de acordo com as orientações dos órgãos de saúde, devido à pandemia de Covid-19. Ocorreu a exibição da obra cinematográfica The Father, seguida de discussão mediada por profissionais de diversas áreas como direito, medicina e gerontologia. Ao final do evento, foi disponibilizado um formulário de presença e avaliação. Resultados: $\mathrm{O}$ tema e a discussão foram considerados relevantes pelos participantes que responderam ao formulário $(\mathrm{N}=19)$. Conclusão: A atividade extensionista favoreceu a interação entre os presentes e a ampliação do olhar sobre as possíveis vivências da terceira idade e da demência, através da arte, prática passível de replicação a outros temas.

Palavras-chave: Demência. Idoso. Obra cinematográfica. Saúde mental.

Abstract: The multidisciplinary event "Cine Saúde" which took place in April of the current year 2021 brought, from a cinematographic work, a starting point to discuss dementia. Objective: To promote a democratic and informative environment to promote expanded dialogue on aging and dementia. Methodology: The event was held on a virtual platform (Google Meet) by the guidelines of the health agencies, due to the Covid-19 pandemic. The

\footnotetext{
${ }^{1}$ Graduanda em Medicina, pela Universidade Estadual do Sudoeste da Bahia, Vitória da Conquista, Bahia, Brasil. Orcid: 0000-0001-6098-0352 E-mail: mariaeduardasgroberto@gmail.com

${ }^{2}$ Graduando em Direito, pela Universidade Estadual do Sudoeste da Bahia, Vitória da Conquista, Bahia, Brasil. Orcid: 0000-0001-7113-3749 E-mail: evans_flavio23@yahoo.com.br

${ }^{3}$ Graduanda em Medicina, pela Universidade Federal da Bahia, Vitória da Conquista, Bahia, Brasil. Orcid: 0000-0001-7396-647X E-mail: gabrielagcl@outlook.com

${ }^{4}$ Graduando em Medicina, pela Universidade Federal da Bahia, Vitória da Conquista, Bahia, Brasil. Orcid: 0000-0001-6709-0678 E-mail: luizpitanga@hotmail.com

${ }^{5}$ Graduanda de Direito, pela Universidade Estadual do Sudoeste da Bahia, Vitória da Conquista, Bahia, Brasil. Orcid: 0000-0003-2423-3100 E-mail: rannasm@ hotmail.com
} 
exhibition of the cinematographic work The Father took place, followed by a discussion mediated by professionals from different areas such as law, medicine, and gerontology. At the end of the event, attendance and evaluation form was made available. Results: The theme and discussion were considered relevant by the participants who answered the form $(N=19)$. Conclusion: The extension activity favored the interaction between those present and the broadening of the view on the possible experiences of the elderly and dementia, through art, a practice that can be replicated to other themes.

Keywords: Cinematographic work. Dementia. Elderly. Mental health.

\section{Introdução}

É notável o processo de envelhecimento no Brasil e no mundo com a progressão da expectativa de vida. Tal processo de envelhecimento é diretamente acompanhado com o aumento das Doenças Crônicas não Transmissíveis (DCNT), sendo, portanto, uma questão de saúde pública de grande importância (SANTOS; BESSA; XAVIER, 2018). A Organização Mundial da Saúde (OMS), desde 2012, reconhece a demência como um problema de saúde pública, visto o significativo impacto socioeconômico e a iminente progressão no número de diagnósticos da doença.

A demência é representada por Freitas (2017) como uma síndrome geriátrica multicausal caracterizada por declínio cognitivo de forma a comprometer significativamente a funcionalidade do paciente. Com isso, os hábitos de vida do paciente como, por exemplo, vestirse, gerenciar as suas finanças, alimentar-se e locomover-se são comprometidos a ponto de o paciente necessitar de auxílio nas atividades de vida diária. O declínio da memória, as alucinações, a agressividade e a alteração de humor promovem não somente a perda cognitiva, mas, principalmente, da própria identidade.

De acordo com Nascimento e Figueiredo (2018, p.1.390), “A demência aparece como uma doença que vai além dos aspectos biológicos, pois afeta o doente e aqueles que lhe estão próximos, principalmente os familiares cuidadores". A partir dessa premissa, é importante salientar os impactos na dinâmica familiar e o grande ônus envolto no processo do cuidar. Por se tratar de uma síndrome de caráter progressivo e sem tratamento capaz de modificar o percurso da doença, nos casos de demência irreversível, todos os atores envolvidos na rede de apoio do paciente sofrem com a sobrecarga de trabalho. Mattos, Oliveira e Novelli (2020, p. 2) destacam que:

Lidar com o declínio das habilidades cognitivas e funcionais da pessoa com demência, oferecer apoio físico e emocional, administrar medicação, lidar com sintomas, com as mudanças de personalidade e perda de comunicação,

Revista Extensão \& Cidadania, v. 9, n. 15, p. 199-208, jan./jun. 2021. 
dentre outras acabam por comprometer a saúde física, emocional, qualidade de vida e bem-estar da pessoa que cuida.

Logo, o processo de atenção à saúde necessita considerar, além das necessidades biológicas, o contexto social em que paciente e rede de apoio se encontram. A progressão da demência alavanca mudanças na realidade sociofamiliar, nas responsabilidades domésticas e legais. Em consequência, evidencia a necessidade do aparato multiprofissional e integral à pessoa idosa e à família. Sobre esse aspecto, o Estatuto do Idoso afirma que:

[...] assegurada a atenção integral à saúde do idoso, por intermédio do Sistema Único de Saúde - SUS, garantindo-lhe o acesso universal e igualitário, em conjunto articulado e contínuo das ações e serviços, para a prevenção, promoção, proteção e recuperação da saúde, incluindo a atenção especial às doenças que afetam preferencialmente os idosos (BRASIL, 2003, Art. 15). As instituições de saúde devem atender aos critérios mínimos para o atendimento às necessidades do idoso, promovendo o treinamento e a capacitação dos profissionais, assim como orientação a cuidadores familiares e grupos de autoajuda. (BRASIL, 2003, Art. 18)

Nesse ínterim, as sucessivas reflexões evidenciam a notoriedade da abordagem de temáticas sobre a pessoa idosa voltada para a importância da transprofissionalidade para o cuidado do idoso com qualquer doença incapacitante. Isto é, os impactos profundos biopsicossociais desencadeados pela síndrome demencial exigem uma visão multifacetada a fim de obter melhor arcabouço de assistência a todos envolvidos no processo do adoecimento e do tratamento.

Assuntos delicados e importantes como a demência exigem um olhar cauteloso e sensível para sua compreensão e debate. O cinema é uma ferramenta chave para discussões, reflexões e sensibilização popular (SÁ; TORRES, 2013). A partir das narrativas, as obras cinematográficas são capazes de promover a exposição de situações-problema, a formação profissional humanizada, além de trazer do mundo das ideias a comunhão entre teoria e prática (DANTAS; MARTINS; MILITÃO, 2010).

Isto posto, os discentes da Liga Acadêmica de Geriatria e Gerontologia (LAGG) e da Liga Acadêmica de Psiquiatria e Saúde Mental (LiAPS) se propuseram a desenvolver a atividade de extensão denominada "Cine Saúde", com exibição da obra cinematográfica The Father, seguida de momento para apreciação e debate sobre as múltiplas facetas da demência. Por conseguinte, objetivou-se a promoção de um ambiente democrático e elucidativo para

Revista Extensão \& Cidadania, v. 9, n. 15, p. 199-208, jan./jun. 2021. 
promoção do diálogo aberto. Dessa forma, com a interação entre acadêmicos, profissionais da saúde, gerontóloga, profissional do Direito e comunidade externa, buscou-se trazer uma discussão mais ampliada e contemplativa sobre a demência.

\section{Metodologia}

O evento "Cine Saúde" foi uma atividade extensionista de caráter pontual, com carga horária de 3 horas, elaborado e executado por meio da parceria entre os discentes da Liga Acadêmica de Geriatria e Gerontologia (LAGG) e a Liga Acadêmica da Psiquiatria e Saúde Mental (LiAPS). O evento concretizou-se no dia 24 de abril de 2021, com início às 15 horas e término às 18 horas. Devido à pandemia de COVID-19 e a necessidade de se manter as normas de biossegurança e o isolamento social, os membros organizadores realizaram o evento por meio da plataforma digital Google Meet. A divulgação do evento ocorreu através das redes sociais do Centro Acadêmico de Medicina Francisca Praguer Fróes (CAMF), da Liga Acadêmica de Geriatria e Gerontologia (LAGG) e da Liga Acadêmica de Psiquiatria e Saúde Mental (LiAPS).

O Cine Saúde exibiu a obra cinematográfica de título The Father. A obra foi dirigida por Florian Zeller, produzida em 2020, tem duração de uma hora e trinta e sete minutos e foi ganhadora do Oscar 2021 nas categorias de melhor roteiro adaptado e melhor ator. Ela conta a história de Anthony - um senhor de idade acometido pela síndrome demencial - que se recusa a receber cuidados e o avanço da doença começa a compromete o bem-estar dele e de toda a família.

Quatro profissionais foram convidados pelas ligas - um da área de Direito, uma gerontóloga, um psiquiatra, uma geriatra - e agruparam de forma independente a discussão acerca do tema proposto, bem como foram abordadas as alterações causadas pela doença, tão somente no paciente e na vida das pessoas próximas, à guisa de exemplo, família, amigos e profissionais da área de saúde.

Após a exibição do filme foi promovida uma discussão sobre os estágios clínicos da doença e os impactos sociais e familiares causados na demência. Ao longo do diálogo, os participantes e os convidados expuseram relatos, opiniões e compreensões sobre a consciência da doença, o conceito, a intervenção do Estado e da família em relação à pessoa idosa.

A ação contou com a presença de 39 participantes, sendo quatro destes são profissionais convidados. Os participantes não necessitaram de inscrição prévia, mas apenas preencher um

Revista Extensão \& Cidadania, v. 9, n. 15, p. 199-208, jan./jun. 2021. 
único formulário de presença disponibilizado via chat ao final do evento para coleta de dados, avaliação do "Cine Saúde" e para emissão dos certificados, com carga horária de três horas - o formulário não contou com a discriminação do público alvo. Dessa forma, o formulário virtual abordava a avaliação do evento, por meio de notas de 1 a 5 (sendo 1 equivalente a péssimo e 5 equivalentes a ótimo), em relação à organização, à programação e à relevância do conteúdo. Para a interpretação dos resultados consideramos a nota 4 como "bom". A avaliação do evento também incluiu uma questão aberta para sugestões, críticas e elogios.

\section{Resultado}

O evento foi iniciado com uma breve apresentação das ligas e da proposta da atividade. Após a exibição do filme, os profissionais convidados apresentaram suas percepções sobre ele, seus personagens e elementos audiovisuais, de forma correlata às relações sociais e de cuidado, bem como às implicações legais que podem se estabelecer diante de um quadro demencial.

As explanações promoveram discussões nas quais os presentes participaram, de forma oral e pelo chat, com perguntas e colocações sobre o filme e a pertinência da temática. Ao final do evento, o formulário para contabilização da presença e apropriação do feedback pela comissão organizadora foi disponibilizado no chat e obteve 19 respostas, apresentadas nas quatro tabelas abaixo. Vejamos:

\section{Tabela 1 - Avaliação da relevância do conteúdo apresentado}

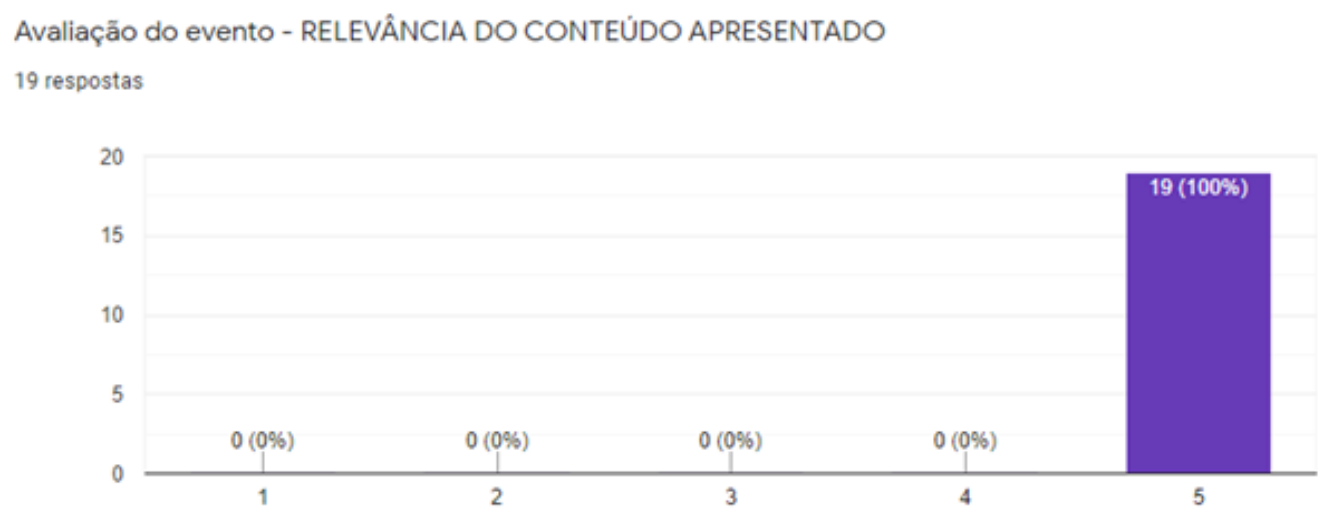

Fonte: Dados da pesquisa. Vitória da Conquista, Bahia, Brasil, 2021 (N=19).

Revista Extensão \& Cidadania, v. 9, n. 15, p. 199-208, jan./jun. 2021.

ISSN 2319-0566 DOI: 10.22481/recuesb.v9i15.8520 


\section{Tabela 2 - Avaliação da pontualidade do evento}

Avaliação do evento - PONTUALIDADE

19 respostas

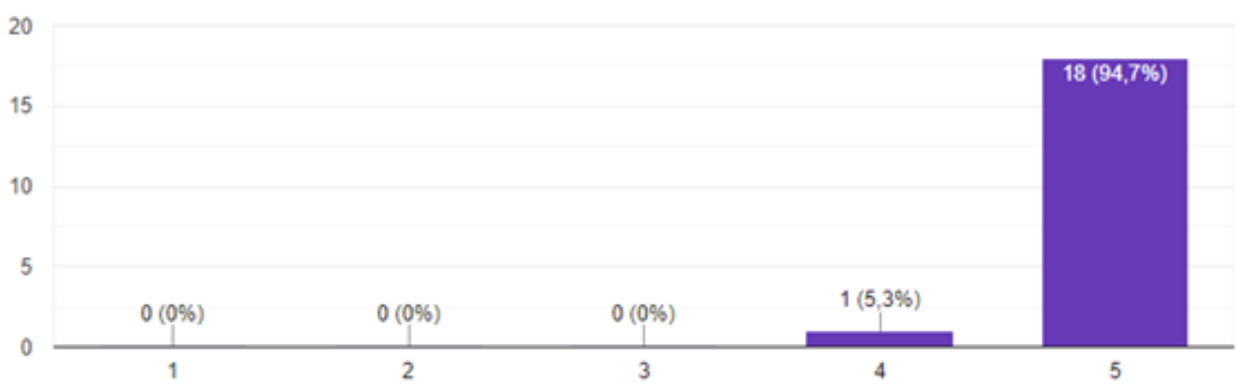

Fonte: Dados da pesquisa. Vitória da Conquista, Bahia, Brasil, 2021 (N=19).

\section{Tabela 3 - Avaliação da organização do evento}

Avaliação do evento - ORGANIZAÇÃO

19 respostas

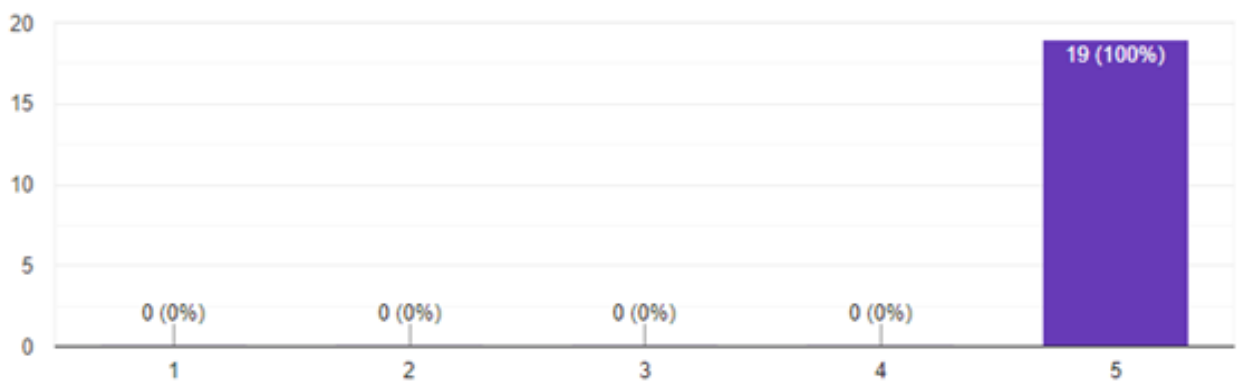

Fonte: Dados da pesquisa. Vitória da Conquista, Bahia, Brasil, 2021 (N=19).

Revista Extensão \& Cidadania, v. 9, n. 15, p. 199-208, jan./jun. 2021.

ISSN 2319-0566 DOI: 10.22481/recuesb.v9i15.8520 


\section{Tabela 4 - Avaliação da programação do evento}

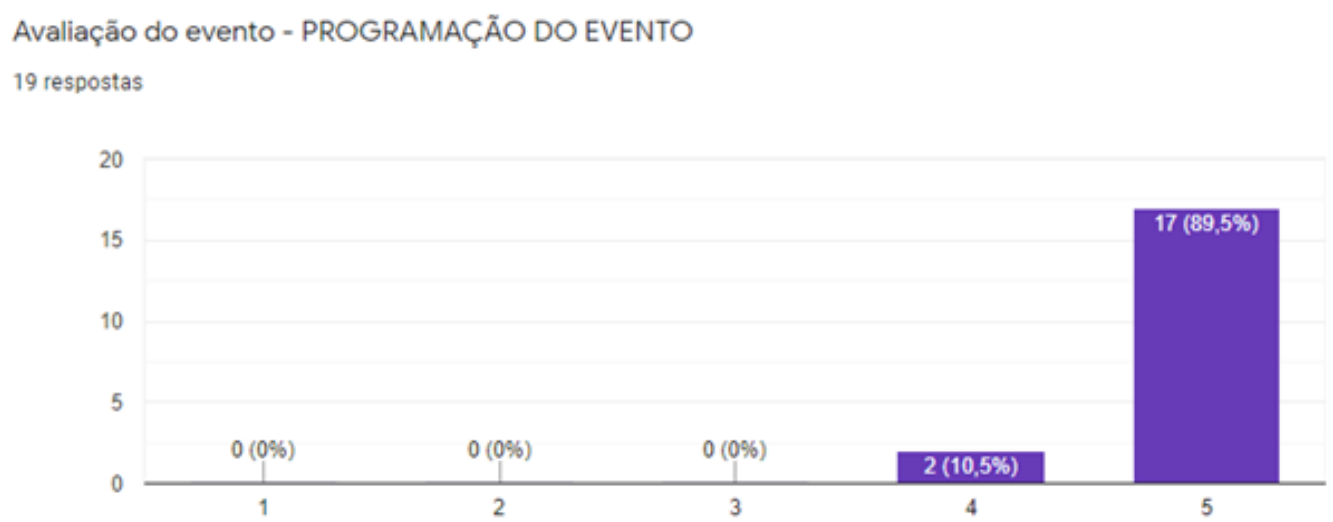

Fonte: Dados da pesquisa. Vitória da Conquista, Bahia, Brasil, 2021 (N=19).

Dessa forma, com relação a programação do evento, 89,5\% das pessoas responderam e avaliaram como ótima e 10,5\% (2 pessoas) como boa. Quanto à pontualidade, 94,5\% a avaliaram como ótima e 5,3\% (1 pessoa) como boa. Já a organização e a relevância do conteúdo foram avaliadas como ótimas em $100 \%$ das respostas.

No espaço discursivo do formulário, foram sugeridos a escolha de outra plataforma para a transmissão, a fim de otimizar a qualidade do áudio, a realização de mais eventos como esse e o tema "incêndios" para um próximo evento. A crítica quanto à qualidade do áudio é compreensível, bem como possíveis quedas de conexão entre os presentes, uma vez que a realização de um evento virtual implica na inclusão de pessoas com acesso a diferentes qualidades de conexão e com diferentes níveis de habilidades tecnológicas.

Apesar dessas dificuldades, elogios ao evento e aos comentários dos convidados se destacaram, bem como solicitações do link do filme através das redes sociais das ligas acadêmicas envolvidas. As avaliações positivas recebidas apontam para a pertinência do evento e da temática, além de reafirmarem a notabilidade e eficácia dele para a construção de um debate efetivo utilizando-se de estratégias lúdicas.

\section{Discussão}

A discussão multidisciplinar promovida pelo evento abrangeu a área do Direito e a gerontologia e, no que tange à saúde, à geriatria e à psiquiatria, contribuindo para a compreensão do idoso em sua esfera biopsicossocial.

Revista Extensão \& Cidadania, v. 9, n. 15, p. 199-208, jan./jun. 2021.

ISSN 2319-0566 DOI: 10.22481/recuesb.v9i15.8520 
O ambiente discursivo sobre o processo de envelhecimento e suas dimensões biológicas, psicológicas e sociais levaram a reflexões acerca do idoso e da sua suscetibilidade à demência. Foram abordadas, ainda, as particularidades no atendimento em saúde mental, principalmente no contexto de uma doença limitante e as relações diretas com os direitos do idoso à residência e à assistência, bem como características morais e éticas que podem causar conflitos consigo mesmo e com pessoas próximas.

Comumente na nossa sociedade, é possível se ter um exemplo de um idoso dependente de cuidados de terceiros. Na ocorrência desta situação concomitantemente a alguma doença, a realidade tanto do idoso como dos seus familiares se altera levando em consideração os extremos cuidados que devem ser tomados em decorrência de uma doença como a demência. Além disso, é recorrente o dilema familiar relativo a interná-lo em uma instituição de longa permanência ou não.

Nesse dilema, é importante esclarecer que a institucionalização não se constitui como crime. Assim como prevê a Lei $\mathrm{n}^{\circ} 10.741$, de $1^{\circ}$ de outubro de 2003 , no artigo 37 , parágrafo $1^{\circ}$ que afirma "A assistência integral na modalidade de entidade de longa permanência será prestada quando verificada inexistência de grupo familiar, casa-lar, abandono ou carência de recursos financeiros próprios ou da família". Contudo, é preciso refletir quanto às perspectivas do idoso e às dimensões de sofrimento psíquico que ele poderá enfrentar. Nessa perspectiva, cabe ponderações sobre demais alternativas complementares ao tratamento, para que se mantenha a qualidade de vida do senescente.

O envelhecimento, assim como exposto por Freitas (2017), se constitui de um processo de mudanças biopsicossociais do ser em si e para si. Essas transformações demandam um acompanhamento do idoso e de toda esfera familiar, social e religiosa que o engloba. Por isso, as consultas médicas e o apoio multiprofissional são imprescindíveis, tanto para um tratamento de saúde, quanto para orientação dos direitos da pessoa idosa e para o aprofundamento dos estudos no campo da gerontologia, especialmente quando há comorbidades envolvidas. Em suma, é imprescindível considerar os direitos e deveres do idoso, a fim de assegurar a proteção, a liberdade, o respeito e a dignidade da pessoa idosa.

\section{Considerações finais}

A arte é uma ferramenta potente para a sensibilização, capaz de promover uma aprendizagem mais significativa. Nessa perspectiva, espaços multidisciplinares de debate que

Revista Extensão \& Cidadania, v. 9, n. 15, p. 199-208, jan./jun. 2021. 
mobilizam e a construção de conhecimento podem favorecer a desconstrução de preconceitos e a expansão da visão sobre a temática. Isso porque o filme, dentre outros recursos artísticos, aproxima determinada realidade da pessoa que o aproveita e com ele pode se identificar.

Sendo a experiência humana, nesse caso do sujeito idoso com demência, complexa e subjetiva, ela envolve valores, comportamentos e contextos com especificidades relativas à idade e à doença, mas com possibilidades plurais. Nessa perspectiva, compreender que, embora o sujeito possa não se lembrar, se confundir, ter seu comportamento alterado, dentre outras manifestações sintomatológicas, ele não se reduz a essas características.

O respeito e o cuidado integral devem, portanto, envolver o reconhecimento de suas limitações a serem manejadas, mas também o reconhecimento de suas possibilidades a serem exploradas visando que desenvolva seu potencial independentemente do contexto - domiciliar, clínico, institucional - em que esse cuidado ocorra.

Por fim, a experiência extensionista evidenciou a importância e a necessidade da realização de ações voltadas para uma discussão ampliada por intermédio de ferramentas lúdicas, o que foi potencializado pela parceria entre ligas voltadas para diferentes especificidades da saúde. A participação multiprofissional amplifica as possibilidades de abordagens e o uso de ferramentas interativas potencializa a participação do público. Logo, urge o estímulo e a divulgação de atividades, capazes de promover um diálogo que aborde a demência de forma multidisciplinar, levando aos participantes de tais eventos, maior compreensão sobre o tema.

\section{Referências}

BRASIL. Lei $\mathbf{n}^{\circ} \mathbf{1 0 . 7 4 1}$, de $1^{\circ}$ de outubro de 2003. Dispõe sobre o Estatuto do Idoso e dá outras providências. Disponível em:

http://www.planalto.gov.br/ccivil_03/leis/2003/110.741.htm. Acesso em: 2 maio 2021.

DANTAS, A. A.; MARTINS, C. H.; MILITAO, M. S. R. O cinema como instrumento cidático para a abordagem de problemas bioéticos: uma reflexão sobre a eutanásia. Revista Brasileira de Educação Médica, Rio de Janeiro, v. 35, n. 1, p. 69-76, mar. 2011.

FREITAS, E.V. et al. Tratado de Geriatria e Gerontologia. 4. ed. Rio de Janeiro: Grupo Editorial Nacional (GEN), 2016.

MATTOS, E. B. T.; OLIVEIRA, J. P.; NOVELLI, M. M. P. C. As demandas de cuidado e autocuidado na perspectiva do cuidador familiar da pessoa idosa com demência. Revista

Brasileira de Geriatria e Gerontologia, Rio de Janeiro, v. 23, n. 3, 2020.

Revista Extensão \& Cidadania, v. 9, n. 15, p. 199-208, jan./jun. 2021.

ISSN 2319-0566 DOI: 10.22481/recuesb.v9i15.8520 
NASCIMENTO, H. G. do; FIGUEIREDO, A. E. B. Demência, familiares cuidadores e serviços de saúde: o cuidado de si e do outro. Ciências, Saúde Coletiva, Rio de Janeiro, v. 24, n. 4, p. 1381-1392, abr. 2019.

OLIVEIRA, I. R. de; ALVES, V. P. A pessoa idosa no contexto da Bioética: sua autonomia e capacidade de decidir sobre si mesma. Revista Kairós Gerontologia, São Paulo, v. 13, n. 2, p. 91-98, nov. 2010.

SÁ, E. C.; TORRES, R. A. T. Cinema como recurso de educação em promoção da saúde. Revista de Medicina, [S. 1.], v. 92, n. 2, p. 104-108, 2013.

SANTOS, C. de S. dos; BESSA, T. A. de; XAVIER, A. J. Fatores associados à demência em idosos. Ciências, Saúde Coletiva, Rio de Janeiro, v. 25, n. 2, p. 603-611, fev. 2020.

WORLD HEALTH ORGANIZATION. Dementia: a public health priority. Suíça, 2012. Disponível em: https://www.alzint.org/u/2020/08/Dementia-A-Public-Health-Priority.pdf. Acesso em: 2 maio 2021.

Recebido: 10.05.2021

Aceito: 19.06 .2021

Revista Extensão \& Cidadania, v. 9, n. 15, p. 199-208, jan./jun. 2021. 